Kotte, E.M.W., Groot, J.F. de, Winkler, A.M.F., Veenhof, C., Takken, T. Fitkids treadmill test: clinical utility and factors associated with its use among physical therapists. Physical Therapy: 2019, 99(4), 428-438

$\begin{array}{ll}\text { Postprint version } & : \\ \text { Journal website } & : \frac{\text { https://academic.oup.com/ptj/advance-article- }}{\text { abstract/doi/10.1093/pti/pzy142/5299592?redirectedFrom=fulltext }} \\ \text { Pubmed link } & : \frac{\text { https://www.ncbi.nlm.nih.gov/pubmed/30690630 }}{\text { DOI }} \\ \text { D.1093/ptj/pzy142 }\end{array}$

This is a Nivel certified Post Print, more info at nivel.nl

\title{
Fitkids Treadmill Test: Clinical Utility and Factors Associated With Its Use Among Physical Therapists
}

\author{
Elles M.W. Kotte, Janke F. de Groot, Alexander M.F. Winkler, Cindy \\ Veenhof, Tim Takken
}

Background. Although the Fitkids Treadmill Test (FTT) has been validated and normative values are available for 6- to 18-year-old children who are healthy, these facts do not automatically imply an uptake of the test in the day-to-day practice of physical therapists. Objective. The objectives of this study were to evaluate the utility of the FTT in different diagnostic groups and to explore potential factors affecting the use of the FTT in clinical practice. Design. Mixed methods with both quantitative and qualitative data were used in this study. Methods. Outcome parameters from the FTT were retrieved from the Fitkids database. For evaluation of the utility of the FTT, 2 indicators, exercise duration and maximal effort, were used. An online survey was sent to physical therapists in Fitkids practices to identify factors affecting the use of the FTT in clinical practice. Results. The percentage of children in each of the diagnostic groups who reached the minimal duration of a maximal exercise test ranged from $94 \%$ to $100 \%$. The percentage of children who reached a peak heart rate of $\geq 180$ beats.per minute ranged from $46 \%$ for children with cognitive, psychological, or sensory disorders to $92 \%$ for children with metabolic diseases. The most important facilitator for the use of the FTT was the fact that most therapists were convinced of the additional value of the FTT. The main barriers were physical therapists' attitudes (resistance to change/lack of experience) and, on the environmental level, the absence of a treadmill ergometer in physical therapist practice. Limitations. Structured interviews would have provided a larger amount of information on potential factors affecting the use of the FTT in clinical practice. Conclusions. This study has shown the clinical utility of the FTT in different diagnostic groups in pediatric physical therapist practice. Responding to the factors identified in this study should enable improved uptake of the FTT in clinical practice.

Comprehensive assessment of aerobic fitness in children, especially those with a medical condition or disability, is an important part of pediatric physical therapy.1 Various valid and reliable treadmill protocols for estimating aerobic fitness are available, but there are questions concerning the utility of most of these protocols in clinical practice by physical therapists in Fitkids practices (ie, physical therapist practices affiliated with the Fitkids Foundation). To overcome these concerns, the Fitkids 
Kotte, E.M.W., Groot, J.F. de, Winkler, A.M.F., Veenhof, C., Takken, T. Fitkids treadmill test: clinical utility and factors associated with its use among physical therapists. Physical Therapy: 2019, 99(4), $428-438$

Treadmill Test (FTT) has been developed.2 The advantages of the FTT over other existing and wellestablished maximal treadmill protocols3-6 are 2-fold. First, the maximal incline of the protocol is restricted to the maximal incline of standard treadmills, which is $15 \%$, as these treadmills are most often available in community physical therapist practices. Second, the protocol starts with $0 \%$ incline making this protocol useful in children and adolescents with a disability or chronic disease. A high incline in the first stage of the protocol often leads to premature exhaustion of the muscles of the lower limbs before achieving cardiac or respiratory limits in this population. Earlier research has shown the FTT to be valid and reproducible for the assessment of aerobic fitness in children and adolescents who are healthy. 2 Also, reference values for 6 - to 18-year-old children who are healthy have been published.7 Although the FFT has been evaluated to be valid and reproducible in children and adolescents who are healthy, these facts do not automatically imply an uptake of the test in the routine day-to-day practice of pediatric physical therapists. Clinical utility is clearly of key concern. Literally, clinical utility refers to "the usefulness of an intervention for, or in, clinical practice." 8 An uptake of the FTT in routine practice implies that therapists should change their established behavior in exercise testing. A widely used approach for understanding the difficulties in changing established behavior in clinical practice is based on a framework of Cabana et al.9 The determinant framework of Cabana et al specifies the barriers and facilitators to the implementation of clinical practice guidelines. In these guidelines, the use of standardized measurement instruments is recommended in order to support clinical decision making. However, the use of these standardized outcome measures is inconsistent in physical therapist practice.10-13 Regarding maximal treadmill protocols, the Bruce treadmill protocol is the most commonly used protocol in clinical practice. In a study regarding the daily practice of exercise testing among pediatric cardiology and pulmonary centers in the United States, it was stated that the (modified) Bruce protocol was used by $79 \% .14$ The modified protocol has smaller increments in workload in comparison with the original Bruce protocol. This value is broadly in line with those in other studies.15 This widespread popularity of the (modified) Bruce protocol is perhaps somewhat surprising, because of the frequently reported disadvantages of the (modified) Bruce protocol, especially in very young children and those with chronic illness.16,17 Before the introduction of the FTT, the (modified) Bruce protocol was also widely used in Fitkids practices However, because of the limited inclination of the available treadmill ergometer in most Fitkids practices, the protocol was often adjusted.

There is only limited knowledge about effective strategies to increase use of outcome measures.13 However, several studies have identified barriers and facilitators to routine use of outcome measures in among physical therapists in clinical practice of which many are similar to those specified by Cabana et al.9,10,12,13,19,20 The barriers identified by Cabana et al are grouped based on whether they affect physician knowledge (lack of awareness and lack of familiarity), attitude, (lack of agreement with (specific) guidelines, skills, motivation, outcome expectancy, self-efficacy, and habits/routines) or behavior. Three external factors that are considered to be related to behavior are the guideline itself, the patient, and social/environmental factors. 9

Identification of facilitators and barriers influencing the use of the FTT among physical therapists in Fitkids practices seems useful for improving uptake of the FTT in the routine day-to-day practice of physical therapists. As the recommendation by the Fitkids Foundation to use the FTT in clinical practice can be interpreted as being a new guideline to monitor aerobic fitness it seems legitimate using the theoretical framework of Cabana et al9 in this identification. Summarized, for the FTT to become well embedded in clinical practice, its utility should be demonstrated in clinical populations and a tailored implementation strategy should be launched based on prospectively identified barriers and facilitators to the use of the FTT in clinical practice. Therefore, the 2 goals of the present study were to evaluate the utility of the FTT in different diagnostic groups (part 1 of the study) and to explore potential factors affecting the use of the FTT in clinical practice (part 2 of the study).

Methods 
Kotte, E.M.W., Groot, J.F. de, Winkler, A.M.F., Veenhof, C., Takken, T. Fitkids treadmill test: clinical utility and factors associated with its use among physical therapists. Physical Therapy: 2019, 99(4), 428-438

FTT

The FTT is an incremental treadmill test consisting of 90-second stages with increments in both speed and grade as described previously.2,7 The test starts after a warming-up period ( $3.5 \mathrm{~km} / \mathrm{h}, 0 \%$ grade) at $3.5 \mathrm{~km} / \mathrm{h}$ and a $1 \%$ gradient, followed by incremental increases in both speed $(0.5 \mathrm{~km} / \mathrm{h})$ and incline (2\%). The maximal incline is $15 \%$. Main outcome measures of the FTT are time to exhaustion (TTE) and peak heart rate (HRpeak). The TTE is calculated as the total duration of the test minus the duration of the warm-up phase.

\section{Knowledge Transfer Activities}

The FTT was developed in close cooperation with physical therapists and pediatric physical therapists in Fitkids practices. Since 2014, the FTT is adopted as one of the core exercise tests outlined in the Fitkids test manual. Concurrently, the validity and the reproducibility of the FTT were investigated and in 2015 the article concerning the validity and reproducibility was published. 2 In 2016 reference values of the FTT in 356 Dutch children who were healthy were published.7 The Fitkids Foundation used the intranet located at our website, social media, and newsletters to inform the therapists about the FTT. The protocol, standard operation procedures and reference values could also be obtained from the intranet and were distributed on several meetings. Different training days and workshops were organized over the past few years to ensure that therapists got familiar with the protocol. The knowledge transfer activities were part of the "Fit for the Future" consortium21 and were based on the policy of the Royal Dutch Society for Physical Therapy, 20 that information about measurement instruments should be disseminated through publication in professional journals, newsletters, and guidelines.

\section{Study Design}

The clinical quantitative data that were used to describe the study population and to evaluate the utility of the FTT in clinical practice were extracted from the Fitkids database. The Fitkids database is a web-based electronic patient record of children participating in the program. Data that were entered between March 2014 and May 2017 were used for this study. Fitkids is a nationwide exercise therapy program in the Netherlands. Fitkids aims to increase health-related fitness, health-related quality of life, and participation in physical activity and/or sports in children and adolescents who are 6 to 18 years old and have a chronic condition or disability.

The data in the database were obtained by physical therapists and pediatric physical therapists during the initial assessment of Fitkids and concerned medical diagnosis, height, weight, HRpeak, and maximal TTE. The medical diagnosis of the children was based on the information provided by the treating physician in the medical referral letter. Body mass index (in $\mathrm{kg} / \mathrm{m} 2$ ) was calculated from weight and height, 2 and standard deviation scores (SDS) were calculated using Dutch growth charts.22 Reference values published in our earlier research7 were used to compare the maximal TTE of children in the present study with that of children who were healthy.

\section{Part 1: Evaluating the Utility of the FTT}

To assess the utility of the FTT in clinical practice, we used 2 indicators. The first was exercise duration (determined as the total duration of the FTT, including the duration of the warm-up phase), in terms of percentage of children and adolescents who reached the minimal duration for a maximal exercise test that was set at 6 minutes for children who were 6 to 12 years old and 8 minutes for those who were 12 years old and older.23 The second was maximal effort, in terms of the percentage of children and adolescents who reached an HRpeak of $\geq 180$ beats.per minute when performing the FTT. The Indicators were considered sufficient when reached by $75 \%$ of the included children in each of the diagnostic groups. 
Kotte, E.M.W., Groot, J.F. de, Winkler, A.M.F., Veenhof, C., Takken, T. Fitkids treadmill test: clinical utility and factors associated with its use among physical therapists. Physical Therapy: 2019, 99(4), 428-438

\section{Part 2: Exploring Potential Factors Affecting the Use of the FTT in Clinical Practice}

To identify barriers and facilitators to the use of the FTT in clinical practice, an online questionnaire was sent to physical therapists in 1 of the 166 Fitkids practices. The survey was sent with the use of Surveymonkey (www.surveymonkey.com). For a solid basis of the survey questionnaire, we used existing questionnaires to formulate questions and create response options.24,25 These questions were supplemented with useful subjects for collecting specific information about the factors affecting the use of the FTT in clinical practice (ie, questions on test characteristics of the FTT). Different types of response categories were used: open-field questions, multiple-choice bullets, visual analog scale (ranging from 0 to 10 ), and binary questions. The survey started with some questions to gather demographic information of the physical therapist. To ensure clarity and assess face validity of the survey items and response options, a draft of the questionnaire was created by E.M.W.K. using the framework of Cabana et al9 to reflect to underlying domains of barriers and facilitators affecting the use of the FTT in daily clinical practice. These domains are knowledge, attitude and external factors. The questionnaire was reviewed and discussed in our research group. Some questions of the survey were answered by all respondents $(1-7,17-20,23,24)$, some were answered by only those who were using the FTT (defined as users) $(8-15,21,22)$, and question 16 was answered only by those who were not using the FTT in clinical practice (defined as nonusers). The Appendix provides more detailed information on the exact content of the online survey. For the purpose of this article, all questions are translated from Dutch to English.

\section{Ethics Issues}

The analysis of the quantitative data was performed on preidentified anonymous patient care data. The data was collected in the course of standard care to evaluate the effects of the Fitkids exercise training program. According to the Dutch Medical Research Involving Human Subjects Act, this study did not require ethics approval. Parents and children gave their written permission to use the data for research purposes.

\section{Data Analysis}

The IBM SPSS Statistics for Windows, version 24.0 (IBM Corp, Armonk, New York), was used for data analysis. For analysis of the quantitative data, the distribution of the data was determined and checked for normal distribution. The medical conditions of the included children were classified according to the diagnostic groups proposed by the American College of Sports and Medicine, 26 to which we have added 1 category, being the category of motor developmental delays. Differences between the maximal TTE of the FTT and reference values were analyzed using the SDS of the TTE, that is, the difference between the observed and predicted values divided by the SD of the reference values. The analysis concerning the evaluation of the utility of the FTT focused on determining whether the indicators were sufficient in each diagnostic group. The analysis of the online survey focused on identifying facilitators and barriers, based on the framework of Cabana et al.9

\section{Role of the Funding Source}

his study was financed by the Johan Cruyff Foundation and the Dutch Foundation Innovation Alliance (SIA-RAAK), part of the Dutch Organization for Scientific Research (NWO) (PRO-4-03). The funding source had no influence on the study's design, conduct, and reporting.

\section{Results}

\section{Study Population}

Data were based on a database export that was done on April 24, 2017. The export file consisted of test results of 415 children ( 260 boys and 155 girls) from 49 Fitkids practices. The median age of the 
Kotte, E.M.W., Groot, J.F. de, Winkler, A.M.F., Veenhof, C., Takken, T. Fitkids treadmill test: clinical utility and factors associated with its use among physical therapists. Physical Therapy: 2019, 99(4), $428-438$

children was 10.7 years (interquartile range: 9.1-12.3), the median body mass index $(\mathrm{kg} / \mathrm{m} 2)$ was 22.4 (interquartile range: 18.3-25.9), and the median body mass index for age score was 2.2 (interquartile range: 0.9-3.1). Because of incomplete datasets, the body mass index scores are based on 275 children. The medical conditions of the included children are outlined in Table 1.

FTT

Initial test results of the included children are presented in Table 2. The median maximal TTE ranged from 9.0 minutes in children with neuromuscular disorders to 11.5 minutes in children with cardiovascular diseases. Compared to the reference values, test results of the FTT showed significantly reduced the maximal TTE for all medical diagnosis groups (median SDS of TTE between -2.1 [in children with metabolic disorders] and -0.8 [in children with cardiovascular diseases]). The FTT was deemed maximal when HRpeak was $\geq 180$ beats.per minute. Median HRpeak ranged from 179 beats.per minute in children with cardiovascular diseases and children with cognitive, psychological or sensory disorders to 197 beats.per minute in children with metabolic diseases.

Part 1: Evaluation of the Utility of the FTT

Overall, $98 \%$ (405/415) of the children reached the minimal duration for a maximal exercise test which was set at 6 minutes for children who were 6 to 12 years old and 8 minutes for those who were 12 years old and older. With respect to maximal effort, 64\% (252/391) of the participants with an HRpeak registration in the database met the objective criteria of maximal effort during the FTT (HRpeak of $\geq 180$ beats.per minute). Table 2 shows the percentages in each of the diagnostic groups.

Part 2: Exploration of Potential Factors Affecting the Use of the FTT in Clinical Practice Of the 166 Fitkids practices in the Netherlands at the time of the online survey, 155 were active. In total, 142 therapists who were involved in exercise testing in children participating in Fitkids completed the online survey from 141 Fitkids practices (response rate: 91\% [141/155]).

Survey respondents. The characteristics of the 142 survey respondents are presented in Table 3. Sixty-three percent of the therapists reported using the FTT in clinical practice.

Knowledge-related factors. In general, therapists were aware of the existence of the FTT. Only 1 respondent explicitly reported not to be aware of the FTT (0.7\%). Users of the FTT rated a mean knowledge score of 7.4 on a scale from 1 to 10 . Fifty-four percent (49/90) of the users reported to have a lot of knowledge of the FTT (score of 8,9 , or 10). Nonusers were not asked to rate their knowledge on a scale from 0 to 10 but were asked for specific knowledge of the FTT by answering 3 questions: What is the main outcome measure of the FTT? Is rail holding permitted? and Are reference values of the FTT available? The users also answered these questions. Ninety-seven percent (87/90) of the users were aware of the main outcome measure of the FTT; the value for nonusers was $56 \%$ (27/48). Ninety-two percent (83/90) of the users reported to know that rail holding is not permitted; the value for nonusers was $73 \%$ (36/49). Ninety-four percent (85/90) of the users reported that they were aware of the existence of the reference values; the value for nonusers was $78 \%$ (38/49).

Attitude-related factors. Ninety-six percent (85/89) of the users were convinced of the additional value of the FTT; the value for nonusers was $62 \%(31 / 50)$. The reasons why the test was found to be of additional value are summarized in Table 4 for both the users and the nonusers. In total, 112 reasons were reported by 79 users and 26 reasons were reported by 22 nonusers. All reasons reported were classified in 5 main categories. With respect to motivation to use the FTT, users rated a mean motivation score of 8.5 on a scale from 1 to 10 , whereas nonusers rated a score of 5.1. With respect to skills in using the FTT, users rated a mean score of 7.5 on a scale from 1 to 10 . Fifty-six percent (50/90) of the therapists reported having good skill in using the FTT (score of 8,9 , or 10$)$, whereas $10 \%(9 / 90)$ of the therapists reported having only a little skill in using the FTT (score of ?35). Nonusers did not answer this question but were assumed to have no skill in using the FTT.

Users were asked whether they found themselves well educated in using the FTT and what their experience is in using the FTT. Seventy-seven percent (69/90) (highly) agreed that they were 
Kotte, E.M.W., Groot, J.F. de, Winkler, A.M.F., Veenhof, C., Takken, T. Fitkids treadmill test: clinical utility and factors associated with its use among physical therapists. Physical Therapy: 2019, 99(4), $428-438$

sufficiently trained in using the FTT in clinical practice. Fifty-seven percent (51/90) reported having a lot of experience in using the FTT. The mean number of FTTs conducted by 27 of these therapists was 41. Both the users and the nonusers were asked whether they were interested in additional training in using the FTT. Of the users, $27 \%(24 / 90)$ reported that they were interested; the value for nonusers was $28 \%(14 / 50)$.

External barriers. Twenty-seven percent (14/52) of the nonusers reported not meeting the practical requirement of having a treadmill ergometer available (either in their practice or at the time of exercise testing). Also, factors related to the measurement instrument itself may hamper the implementation of the FTT. Therefore, we asked whether the FTT was found to be time-consuming to conduct. Sixteen percent (14/90) of the users agreed with the statement "conducting the FTT is timeconsuming." As therapists who found the FTT highly demanding for children with a medical condition are less likely to use the FTT, we stated that "the FTT is a highly demanding physical test for children who participate in the Fitkids program." Of the users, 7\% (6/90) (highly) agreed with this statement. Pediatric physical therapists are also less likely to use the FTT in clinical practice if they assume that children who are visiting the practice are not able to reach their maximal effort on the FTT. Of the users, $32 \%$ (29/90) disagreed with the statement that "children often reach their maximal effort when performing the FTT." The users who disagreed with the statement reported the following reasons for limiting maximal effort. In total, 61 reasons were reported by 29 therapists: the FTT is difficult because holding onto the front handrail during the test is not permitted $(38 \% ; 23 / 61)$, painful legs $(31 \%$; $19 / 61)$, walking on the treadmill with increments in speed and inclination is too difficult (18\%; 11/61), motivational problems/lack of perseverance $(10 \% ; 6 / 61)$ and the physiological response to (maximal) aerobic exercise is unfamiliar or unknown to children $(3 \% ; 2 / 61)$.

Reasons for not using the FTT in clinical practice are summarized in Table 5 . In total, 44 nonusers reported a reason and $50 \%(22 / 44)$ of the reasons reported could be classified in the category attitude related, of which $39 \%(17 / 44)$ were related to resistance to change (habits) and $11 \%(5 / 44)$ were related to lack of experience. The other $50 \%$ of the reasons reported were related to external barriers, which could be classified into 3 main categories: patient $(9 \% ; 4 / 44)$, organization (39\%; 17/44), and measurement related $(2 \% ; 1 / 44)$. There were no reasons reported that could be classified in the category knowledge related.

Table 6 provides an overview of factors that seemed to have influenced the implementation of the FTT in clinical practice.

\section{Discussion}

The objectives of the study were to evaluate the utility of the FTT in different diagnostic groups and to explore potential factors affecting the use of the FTT in clinical practice. The test results of the FTT showed significantly reduced median maximal TTE for all diagnostic groups (SDS ranging from -2.1 to -0.8 ), indicating sufficient discriminatory ability of the FTT. The findings of reduced aerobic fitness levels in children participating in Fitkids are consistent with the findings in our previous studies.18,27

\section{Part 1: Evaluation of the Utility of the FTT}

The first indicator to evaluate the utility of the FTT was successful as the minimal exercise duration of 6 minutes in children (6-12 years old) and 8 minutes in adolescents ( 12 years old and older) was reached in more than $75 \%$ of the included children in each diagnostic group. It is important to well inform the therapists on the minimal duration of a maximal cardiorespiratory exercise test. Maximal cardiopulmonary exercise tests with extremely short durations should be interpreted with caution and used evaluative as the oxygen uptake may not have been reached its maximum. The second indicator, to evaluate utility, was successful if $75 \%$ of the children in each diagnostic group reached an HRpeak of $\geq 180$ beats.per minute. This indicator was, however, only reached in children with metabolic diseases $(92 \%)$ and neuromuscular diseases (82\%). In fact, $36 \%$ of the participants with an HRpeak registration 
Kotte, E.M.W., Groot, J.F. de, Winkler, A.M.F., Veenhof, C., Takken, T. Fitkids treadmill test: clinical utility and factors associated with its use among physical therapists. Physical Therapy: 2019, 99(4), $428-438$

in the database had an HRpeak below 180 beats.per minute. From the online survey, we learned that $68 \%$ of the therapists who are using the FTT in clinical practice found the test feasible on HRpeak as they agreed with the statement that "children often reach their maximal effort when performing the FTT." It is known from literature that, for instance, children with mental retardation have lower predicted HRpeak.28 The recommended lower limit of normal for HRpeak of 180 beats.per minute to identify maximal effort seems therefore not applicable in this population. Moreover, in our study on reference values of the FTT, 356 Dutch children who were healthy were included and a lower limit of normal of 174 beats.per minute was found in girls (the mean HRpeak \pm SD values in boys and girls were $197 \pm 7$ and $198 \pm 7$ beats.per minute, respectively). 7 Finally, some children might have problems with movement coordination or have, for instance, an equinus position of the foot. A maximal treadmill test is likely to be symptom-limited in these children resulting in a symptom-limited HRpeak. There are other validated field tests that better suits these children.29-31 We advise therapists to choose measurement instruments that are tailored to the individual child and to use subjective symptoms for maximal effort, such as sweating, facial blushing, and a clear unwillingness to continue despite encouragement. Moreover, as it is questioned whether therapists push and encourage children enough to reach their maximal effort during a maximal exercise test we aim at training therapists in conducting maximal exercise tests.

From the online survey, it became clear that there are 3 main reasons for limiting maximal effort during the FTT: the test is difficult because holding onto the front handrail during the test is not permitted, painful legs, and walking on a treadmill machine with increments in speed and inclination is too difficult. These reasons are likely to be related to being not familiar with treadmill walking. To ensure the opportunity for a successful evaluation, children must be familiarized with the testing procedure.16 With respect to the FTT, it is essential that children are familiar with walking and running on a treadmill machine and with an $15 \%$ inclination angle before the child performs the FTT. On the basis of the current findings, we advise therapist to conduct 2 FTTs on 2 different sessions, as is also recommended for other field walking tests. 32 Ideally, we recommend a practice session before the actual test. In the context of Fitkids, we recommend performing a practice session during the Fitkids lesson in the week before the actual test session is planned. We are aware, however, that this might not always be feasible in clinical practice.

\section{Part 2: Exploration of Potential Factors Affecting the Use of the FTT in Clinical Practice}

A substantial percentage of therapists in Fitkids practices (63\%) have adapted their established behavior in exercise testing. The percentage of therapists using the FTT in clinical practice approaches the percentage of those using the (modified) Bruce treadmill test in the clinical practice of pediatric cardiology and pulmonary centers in the United States (79\%). However, we are aware of the fact that therapists in Fitkids practices are a demarcated population of therapists in the Netherlands and that the use of the FTT in general is much lower. The online survey yielded information on factors we can respond to improve uptake of the FTT in the routine day-to-day practice of pediatric physical therapists.

\section{Discussion of Factors Influencing the Use of the FTT on the Basis of Factors Proposed by Cabana et al9}

The online survey revealed a high level of awareness of the FTT among physical therapists in Fitkids practices and revealed that the knowledge of the FTT among the users was high. It can be concluded that the strategy to improve knowledge as used by the Fitkids Foundation was quite successful. However, the nonusers revealed to have far less detailed knowledge on the FTT and this might have contributed to the fact that these therapists do not use the FTT yet. Important reasons that were reported for not using the FTT although being convinced of the additional value were, attitude related, resistance to change or habits and at the organizational level the absence of a treadmill ergometer. It 
Kotte, E.M.W., Groot, J.F. de, Winkler, A.M.F., Veenhof, C., Takken, T. Fitkids treadmill test: clinical utility and factors associated with its use among physical therapists. Physical Therapy: 2019, 99(4), $428-438$

seems likely that therapists who cannot use the test because of the absence of a treadmill ergometer are less interested in the provided information on the FTT and therefore have less detailed knowledge. From literature it is known that habits cause resistance to change at the individual level.33 Miller et al34 investigated the influence of providing information about a change on resistance to change and concluded that detailed "quality" information about a change minimizes resistance at the individual level. Keeping this in mind, still a lot can be achieved by distributing detailed information on the FTT in the population of therapists that are not using the FTT in clinical practice yet. Currently, we are working on an integrated digital knowledge transfer system with information, detailed protocols, and information videos to more widely distribute information on the FTT.

Logically, the nonusers were less motivated to use the FTT in clinical practice. Next to habits, a lower level of motivation is also known to cause resistance to change at the individual level. 33 Therefore, it is of interest which internal or external factors may help to motivate therapists who are nonusers to use the FTT. The fact that the nonusers lack skills in using the FTT may result in a low motivation, because of lack of self-efficacy. Self-efficacy refers to the belief of being capable of carrying out a specific task (ie, conducting the FTT). An individual's sense of capability is strongly related to his perception, motivation, and performance as people rarely attempt to perform a task when they expect to be unsuccessful.35 Appropriate educational workshops or training days would raise practical skills and maybe motivate therapist in using the FTT among the nonusers and this might facilitate further use of the FTT in clinical practice. Training days will also facilitate further use as it will help those therapists who reported not to use the FTT because of lack of experience.

The most dominant, attitude-related facilitator for use of the FTT in clinical practice seems the fact that most therapists $(83 \% ; 116 / 139)$ indicated being convinced of the added value of the FTT to the quality of exercise testing in clinical practice. This is related to the fact that the FTT was developed in close cooperation with the therapists. More than half of the nonusers (62\%) indicated being convinced of the additional value of the FTT. Both in the users as the nonusers, the maximal incline of $15 \%$ was mentioned as the most important reason for the FTT being of added value. Part of the therapists may have reported this reason out of practical considerations because the test can be conducted on a standard treadmill ergometer), whereas others may have reported this reason because they found a maximal gradient of $15 \%$ sufficient for the target group. Midgley et al36 recommend that treadmill tests should not surpass $15 \%$ degree in inclination. Although the reason for this recommendation remains more or less unclear, the recommendation may come from the fact that intolerable treadmill grades may limit the ability to elicit a maximal cardiopulmonary effort.

With respect to external factors, it can be concluded that the test characteristics of the FTT might have facilitated the use of the FTT in clinical practice, as only a small minority found the test timeconsuming (16\%) and highly demanding for children with a medical condition or disability (7\%). The FTT is a valid and reliable test with normative values available that can be executed on a standard treadmill machine, starting with a $0 \%$ incline. As there is no such test available like the FTT it may be concluded that the superiority of the FTT to other maximal treadmill protocols also have facilitated the use of the test in clinical practice. Moreover, it is likely to assume that the commitment of the therapists to the Fitkids Foundation has contributed to the fact that the FTT is already being used by $63 \%$. The FitkidsFoundation had developed the FTT, and because of the commitment between the Foundation and Fitkids practices, physical therapists in Fitkids practices are more likely to use the FTT in clinical practice. Moreover, the Fitkids Foundation and the nearly 170 affiliated Fitkids practices can be interpreted as being a social network. There is a significant relationship between individuals' attitudes during a recently implemented change and the attitudes of others in their network.37 Increased use of the FTT outside Fitkids practices would therefore be a bit more complicated because of lack of this commitment and/or lack of a social network. Finally, the fact that usage of the FTT does not entail additional costs is likely to have facilitated the use of the test in clinical practice. 
Kotte, E.M.W., Groot, J.F. de, Winkler, A.M.F., Veenhof, C., Takken, T. Fitkids treadmill test: clinical utility and factors associated with its use among physical therapists. Physical Therapy: 2019, 99(4), 428-438

\section{Limitations}

An online survey was used to identify factors influencing the use of the FTT in clinical practice. Using structured interviews would have allowed us to continue asking about underlying thoughts and would have provided us with a larger amount of information. Moreover, clinical utility is a much broader multi-dimensional judgment including usefulness, benefits and drawbacks of a new intervention. This study mainly focused on the clinical usefulness of the FTT. Furthermore, the narrow geographical location of the Netherlands in which the study data were collected might also be a limitation of this study. Finally, the results on the utility of the FTT in children with cardiovascular diseases should be interpreted with caution as these results are based on only 2 children.

\section{CONCLUSIONS}

The clinical utility of the FTT has been demonstrated in children with neuromuscular and metabolic diseases. In general, the FTT seems also useful in the remaining diagnostic groups of this study. However, prior to testing, it should always be questioned whether the individual child is able to reach for his or her maximal effort during the FTT. Therapists should be trained in conducting maximal exercise tests and children should be familiar with the testing procedure to enable the opportunity of successful evaluations. Only successful evaluations should be interpreted in clinical practice. The online survey revealed important factors to the use of the FTT in clinical practice. Responding to the factors identified in this study enables an improved uptake the FTT in clinical practice.

Disclosure

The authors completed the ICJME Form for Disclosure of Potential Conflicts of Interest. They reported no conflicts of interest.

\section{REFERENCES}

1. Rowland JL, Fragala-Pinkham M, Miles C, O’Neil ME. The scope of pediatric physical therapy practice in health promotion and fitness for youth with disabilities. Pediatr Phys Ther. 2015;27:2-15.

2. Kotte EM, de Groot JF, Bongers BC, Winkler AM, Takken T. Validity and reproducibility of a new treadmill protocol: the Fitkids Treadmill Test. Med Sci Sports Exerc. 2015;47:2241-2247.

3. van der Cammen-van Zijp MH, van den Berg-Emons RJ, Willemsen SP, Stam HJ, Tibboel D, IJsselstijn $\mathrm{H}$. Exercise capacity in Dutch children: new reference values for the Bruce treadmill protocol. Scand J Med Sci Sports. 2010;20:e130-e136.

4. Balke B, Ware RW. An experimental study of physical fitness of air force personnel. U S Armed Forces Med J. 1959;10:675-688.

5. Hollenberg M, Ngo LH, Turner D, Tager IB. Treadmill exercise testing in an epidemiologic study of elderly subjects. J Gerontol A Biol Sci Med Sci. 1998;53:B259-B267.

6. Dubowy KO, Baden W, Bernitzki S, Peters B. A practical and transferable new protocol for treadmill testing of children and adults. Cardiol Young. 2008;18:615-623.

7. Kotte EM, de Groot JF, Bongers BC, Winkler AM, Takken T. Fitkids Treadmill Test: age- and sexrelated normative values in Dutch children and adolescents. Phys Ther. 2016;96:1764-1772.

8. Smart A. A multi-dimensional model of clinical utility. Int J Qual Health Care. 2006;18:377-382. 9. Cabana MD, Rand CS, Powe NR, et al. Why don't physicians follow clinical practice guidelines? A framework for improvement. JAMA. 1999;282:1458-1465.

10. Swinkels RA, van Peppen RP, Wittink H, Custers JW, Beurskens AJ. Current use and barriers and facilitators for implementation of standardized measures in physical therapy in the Netherlands. BMC Musculoskelet Disord. 2011;12:106.

11. Van Peppen RP, Maissan FJ, Van Genderen FR, Van Dolder R, Van Meeteren NL. Outcome measures in physiotherapy management of patients with stroke: a survey into self-reported use, and barriers to and facilitators for use. Physiother Res Int. 2008;13:255-270. 
Kotte, E.M.W., Groot, J.F. de, Winkler, A.M.F., Veenhof, C., Takken, T. Fitkids treadmill test: clinical utility and factors associated with its use among physical therapists. Physical Therapy: 2019, 99(4), $428-438$

12. Jette DU, Halbert J, Iverson C, Miceli E, Shah P. Use of standardized outcome measures in physical therapist practice: perceptions and applications. Phys Ther. 2009;89:125-135.

13. Käll I, Larsson ME, Bernhardsson S. Use of outcome measures improved after a tailored implementation in primary care physiotherapy: a prospective, controlled study. J Eval Clin Pract. 2016;22:668-676.

14. Chang RR, Gurvitz M, Rodriguez S, Hong, E, Klitzner TS. Current practice of exercise stress testing among pediatric cardiology and pulmonary centers in the United States. Pediatr Cardiol. 2006;27:110116.

15. Myers J, Bellin D. Ramp exercise protocols for clinical and cardiopulmonary exercise testing. Sports Med. 2000;30:23-29.

16. Takken T, Blank AC, Hulzebos EH, van Brussel M, Groen WG, Helders PJ. Cardiopulmonary exercise testing in congenital heart disease: equipment and test protocols. Neth Heart J. 2009;17:339-344. 17. Duff DK, de Souza AM, Human DG, Potts JE, Harris KC. A novel treadmill protocol for exercise testing in children: the British Columbia children's hospital protocol. BMJ Open Sports Exerc Med. 2017;3:e000197.

18. Kotte EM, Winkler AM, Takken T. Fitkids exercise therapy program in the Netherlands. Pediatr Phys Ther. 2013;25:7-13.

19. El-Sobkey SB, Helmy AM. Evidence-based practice and standardized outcome measures: Egyptian physical therapists' belief, perceptions and adoption. World Appl Sci J. 2012;16:1282-1291.

20. Stevens JG, Beurskens AJ. Implementation of measurement instruments in physical therapist practice: development of a tailored strategy. Phys Ther. 2010;90:953-961.

21. de Groot JF. Clinical spotlight: Fit for the Future! researchers, (future) physical therapist and families working together towards healthy active lifestyles for children with chronic disease or childhood disability. The IOPTP Newsletter. 2016;16:11-13.

22. alma , ch nbeck , Bakker B. ira ing A, van Buuren . Groeidiagrammen 2010 Handleiding bij het Meten en Wegen van Kinderen en het Invullen van Groeidiagrammen. Leiden, the Netherlands: TNO; 2010.

23. Hebestreit H. Exercise testing in children: what works, what doesn't, and where to go? Paediatr Respir Rev. 2004;5(suppl A):S11-S14.

24. Pisters MF, Leemrijse CJ. Het gebruik van aanbevolen meetinstrumenten in de fysiotherapiepraktijk: meten is nog geen weten! [The use of measurement instruments in physiotherapy practice: knowing it isn't measuring it!]. Ned Tijdschrift Fysiother. 2007;117:176-181. 25. Grol R, Wensing M. Effective implementation: a model. In: Grol R, Wensing M, Eccles M, eds. Improving Patient Care: The Implementation of Change in Clinical Practice. London, United Kingdom: Elsevier; 2005. 26. Durstine JL, Moore GE, Painter PL, et al, eds. ACSM's Exercise Management for Persons with Chronic Diseases and Disabilities. 3rd ed. Champaign, IL: Human Kinetics, 2009:175-181. 27. Kotte EM, de Groot JF, Winkler AM, Huijgen BC, Takken T. Effects of the Fitkids exercise therapy program on health-related fitness, walking capacity, and health-related quality of life. Phys Ther. 2014;94:1306-1318.

28. Fernhall B, McCubbin JA, Pitetti KH, et al. Prediction of maximal heart rate in individuals with mental retardation. Med Sci Sports Exerc. 2001;33:1655-1660.

29. Verschuren O, Takken T, Ketelaar M, Gorter JW, Helders PJ. Reliability and validity of data for 2 newly developed shuttle run tests in children with cerebral palsy. Phys Ther. 2006;86:1107-1117. 30. de Groot JF, Takken T, de Graaff S, Gooskens RH, Helders PJ, Vanhees L. Treadmill testing in ambulatory children with spina bifida: does peak oxygen uptake reflect maximum oxygen uptake? Phys Ther. 2009;89:679-697.

31. de Groot JF, Takken T, Gooskens RH, et al. Reproducibility of maximal and submaximal exercise testing in 'normal ambulatory' and 'community ambulatory' children and adolescents with spina bifida: which is best for the evaluation and application of exercise training? Phys Ther. 2011;91:267276. 
Kotte, E.M.W., Groot, J.F. de, Winkler, A.M.F., Veenhof, C., Takken, T. Fitkids treadmill test: clinical utility and factors associated with its use among physical therapists. Physical Therapy: 2019, 99(4), 428-438

32. Holland AE, Spruit MA, Troosters T, et al. An official European Respiratory Society/American Thoracic Society technical standard: field walking tests in chronic respiratory disease. Eur Respir J. 2014;44:1428-1446.

33. Bennebroek Gravenhorst K. A different view on resistance to change. Paper presented at: "Power Dynamics and Organizational Change IV" EAWOP Conference; May 14-17, 2003; Lisbon, Portugal. 34. Miller VD, Johnson JR, Grau J. Antecedents to willingness to participate in a planned organizational change. J Appl Commun Res. 1994;22:59-80. 35. Bandura, A. Self-Efficacy: The Exercise of Control. New York, NY: Freeman and Co; 1997.

36. Midgley AW, Bentley DJ, Luttikholt H, McNaughton LR, Millet GP. Challenging a dogma of exercise physiology: does an incremental exercise test for valid VO2 max determination really need to last between 8 and 12 minutes? Sports Med. 2008;38:441-447.

37. Burkhardt ME. Social interaction effects following a technological change: a longitudinal investigation. Acad Manage J. 1994;37:869-898. 


\section{Table 1.}

Classification of Primary Medical Diagnoses According to the American College of Sports Medicine $^{a}$

\begin{tabular}{|c|c|c|}
\hline Classification & Diagnosis & $\begin{array}{l}\text { Total No. } \\
\text { (\%) of } \\
\text { Participants }\end{array}$ \\
\hline Cardiovascular diseases & Congenital heart defects & $2(0.5)$ \\
\hline Pulmonary diseases & Asthma, cystic fibrosis & $36(9)$ \\
\hline Metabolic diseases & Diabetes & $13(3)$ \\
\hline $\begin{array}{l}\text { Immunological and hematological } \\
\text { disorders }\end{array}$ & $\begin{array}{l}\text { Chronic fatigue syndrome, } \\
\text { cancer, abdominal organ } \\
\text { transplant, juvenile arthritis }\end{array}$ & $18(4)$ \\
\hline Musculoskeletal/orthopedic disabilities & $\begin{array}{l}\text { Hypermobility syndrome, } \\
\text { toeing-in/toeing-out, fractures }\end{array}$ & $64(15)$ \\
\hline Neuromuscular disorders & $\begin{array}{l}\text { Cerebral palsy, spina bifida, Erb } \\
\text { palsy, epilepsy, ataxia }\end{array}$ & $31(8)$ \\
\hline $\begin{array}{l}\text { Cognitive, psychological, and sensory } \\
\text { disorders }\end{array}$ & $\begin{array}{l}\text { Autism spectrum disorders, } \\
\text { ADHD, mental retardation, } \\
\text { headache, hyperventilation, } \\
\text { sensory processing disorder }\end{array}$ & $94(23)$ \\
\hline Motor developmental delays and DCD & & $147(35)$ \\
\hline Unexplained condition & & $10(2)$ \\
\hline
\end{tabular}

${ }^{a} \mathrm{ADHD}=$ attention-deficit/hyperactivity disorder; $\mathrm{DCD}=$ developmental coordination disorder. 


\section{Table 2.}

Initial Test Results for the $\mathrm{FTT}^{a}$

\begin{tabular}{|c|c|c|c|c|}
\hline Classification & $\begin{array}{l}\text { Maximal TTE } \\
(\min )^{b}\end{array}$ & $\begin{array}{c}\text { SDS of maximal } \\
\text { TTE }^{b}\end{array}$ & $\begin{array}{c}\mathrm{HR}_{\text {peak }} \\
\left(\text { Beats } \cdot \min ^{-1}\right)^{b}\end{array}$ & $\begin{array}{c}\text { \% of } \\
\text { Participants } \\
\text { Who Reached } \\
\text { Minimal } \\
\text { Exercise } \\
\text { Duration }{ }^{c}\end{array}$ \\
\hline Cardiovascular diseases & $11.5^{e}$ & -0.8 & 179 & 100 \\
\hline Pulmonary diseases & 9.7 (9.0 to 10.5$)$ & $\begin{array}{c}-1.3(-2.3 \text { to } \\
-0.5)\end{array}$ & $190(178 \text { to } 197)^{f}$ & 97 \\
\hline Metabolic diseases & 9.5 (7.7 to 10.8$)$ & $\begin{array}{c}-2.1(-3.9 \text { to } \\
-0.6)\end{array}$ & 197 (188 to 200 ) & 100 \\
\hline $\begin{array}{l}\text { Immunological and } \\
\text { hematological disorders }\end{array}$ & $\begin{array}{c}10.5 \text { (9.0 to } \\
11.5)\end{array}$ & $\begin{array}{c}-1.3(-3.5 \text { to } \\
0.2)\end{array}$ & 187 (176 to 194$)$ & 100 \\
\hline $\begin{array}{l}\text { Musculoskeletal/orthopedic } \\
\text { disabilities }\end{array}$ & 9.2 (8.2 to 11.0$)$ & $\begin{array}{c}-1.7(-2.7 \text { to } \\
-0.2)\end{array}$ & $180(168 \text { to } 193)^{g}$ & 100 \\
\hline Neuromuscular disorders & $9.0(7.5$ to 10.3$)$ & $\begin{array}{c}-1.9(-4.1 \text { to } \\
-0.8)\end{array}$ & $187(180 \text { to } 194)^{h}$ & 97 \\
\hline $\begin{array}{l}\text { Cognitive, psychological, } \\
\text { and sensory disorders }\end{array}$ & 9.3 (8.3 to 10.5$)$ & $\begin{array}{c}-1.8(-3.2 \text { to } \\
-1.0)\end{array}$ & $179(164 \text { to } 191)^{i}$ & 94 \\
\hline Motor developmental & 9.7 (8.5 to 11.0$)$ & $-1.5(-2.9$ to & $188(178 \text { to } 196)^{j}$ & 99 \\
\hline
\end{tabular}




\begin{tabular}{|l|l|l|l|l|}
\hline delays and DCD & $0.0)$ & & \\
\hline
\end{tabular}

${ }^{a} \mathrm{DCD}=$ developmental coordination disorder $; \mathrm{FTT}=$ Fitkids Treadmill Test; $\mathrm{HR}_{\text {peak }}=$ peak heart rate; TTE = time to exhaustion.

${ }^{b}$ Values are given as medians (interquartile ranges).

${ }^{c}$ The minimal duration for a maximal exercise test was set at 6 minutes for children who were 6-12 years old and 8 minutes for those who were 12 years old and older.

${ }^{d}$ Defined as an $H R_{\text {peak }}$ of $\geq 180$ beats $\cdot \min ^{-1}$.

${ }^{e}$ Based on $\mathrm{n}=2$.

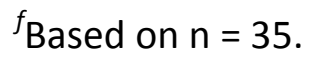

${ }^{g}$ Based on $\mathrm{n}=62$.

${ }^{h}$ Based on $\mathrm{n}=29$.

'Based on $n=87$.

${ }^{j}$ Based on $n=135$. 
Table 3.

Characteristics of Survey Respondents $(n=142)$

\begin{tabular}{|c|c|c|}
\hline \multirow[t]{2}{*}{ Characteristic } & \multicolumn{2}{|c|}{ No. (\%) of: } \\
\hline & Users $(n=90)$ & Nonusers $(n=52)$ \\
\hline \multicolumn{3}{|l|}{ Sex } \\
\hline$M$ & $11(12)$ & $7(14)$ \\
\hline $\mathrm{F}$ & $79(88)$ & $45(87)$ \\
\hline \multicolumn{3}{|l|}{ Age, y } \\
\hline $21-25$ & $6(7)$ & $3(6)$ \\
\hline $25-35$ & $47(52)$ & $24(46)$ \\
\hline $35-45$ & $21(23)$ & $7(14)$ \\
\hline $45-55$ & $9(10)$ & $12(23)$ \\
\hline$>55$ & $7(8)$ & $6(12)$ \\
\hline \multicolumn{3}{|l|}{ Working experience, $y$} \\
\hline $0-2$ & $4(4)$ & $3(6)$ \\
\hline $2-4$ & $6(7)$ & $3(6)$ \\
\hline $4-6$ & $8(9)$ & $3(6)$ \\
\hline $6-8$ & $14(16)$ & $7(13)$ \\
\hline $8-10$ & $20(22)$ & $7(13)$ \\
\hline$>10$ & $38(42)$ & $29(56)$ \\
\hline \multicolumn{3}{|l|}{ Profession } \\
\hline Pediatric physical therapist & $67(74)$ & $45(87)$ \\
\hline
\end{tabular}




\begin{tabular}{|c|c|c|}
\hline Pediatric physical therapist & $11(12)$ & $1(2)$ \\
in training & & $6(12)$ \\
\hline General physical therapist & $12(13)$ & \\
\hline Experience in using & & $11(21)$ \\
\hline $0-2$ & $28(31)$ & $11(21)$ \\
\hline $2-4$ & $17(19)$ & $10(19)$ \\
\hline $4-6$ & $17(19)$ & $20(39)$ \\
\hline$>6$ & $28(31)$ & \\
\hline
\end{tabular}




\section{Table 4.}

Reasons Why Therapists Found the FTT of Added Value ${ }^{a}$

\begin{tabular}{|l|c|c|}
\hline Reason & \multicolumn{2}{|c|}{ No. (\%) of reasons } \\
\cline { 2 - 4 } & Users & Nonusers \\
(n=22)
\end{tabular}

${ }^{a}$ FTT $=$ Fitkids Treadmill Test. 


\section{Table 5.}

Reasons Reported for Not Using the FTT in Clinical Practice ${ }^{a}$

\begin{tabular}{|c|c|}
\hline Classification & $\begin{array}{l}\text { No. (\%) of } \\
\text { Nonusers } \\
(n=44)\end{array}$ \\
\hline \multicolumn{2}{|l|}{ Attitude related } \\
\hline Resistance to change (habits) & $17(39)$ \\
\hline Lack of experience & $5(11)$ \\
\hline \multicolumn{2}{|l|}{ External barrier } \\
\hline \multicolumn{2}{|l|}{ Patient } \\
\hline Different preferences & $1(2)$ \\
\hline $\begin{array}{l}\text { Child cannot be tested because the test is too } \\
\text { demanding }\end{array}$ & $1(2)$ \\
\hline $\begin{array}{l}\text { Contraindications to maximal exercise } \\
\text { (cardiovascular problems, exercise intolerance) }\end{array}$ & $2(5)$ \\
\hline \multicolumn{2}{|l|}{ Organization } \\
\hline No treadmill machine available & $14(32)$ \\
\hline No time for individual testing & $3(7)$ \\
\hline \multicolumn{2}{|l|}{ Measurement instrument } \\
\hline Test is time-consuming & $1(2)$ \\
\hline
\end{tabular}

${ }^{a}$ FTT $=$ Fitkids Treadmill Test. 
Table 6.

Overview of Factors Important in the Implementation of the $\mathrm{FTT}^{a}$

\begin{tabular}{|c|c|c|}
\hline Factor & $\begin{array}{l}\text { Users } \\
(n=90)\end{array}$ & $\begin{array}{l}\text { Nonusers } \\
(n=52)\end{array}$ \\
\hline \multicolumn{3}{|l|}{ Knowledge related } \\
\hline Awareness of the FTT & $100 \%$ & $98 \%$ \\
\hline Mean knowledge score & 7.4 & \\
\hline $\begin{array}{l}\text { Respondents who correctly answered } 3 \\
\text { questions related to the FTT }\end{array}$ & $84 \%$ & $44 \%^{b}$ \\
\hline \multicolumn{3}{|l|}{ Attitude related } \\
\hline Convinced of added value & $96 \%^{c}$ & $62 \%{ }^{d}$ \\
\hline Mean motivation score & 8.5 & 5.1 \\
\hline Good skills in using the FTT & $56 \%$ & $\begin{array}{c}\text { Assumed to } \\
\text { have no } \\
\text { skills }\end{array}$ \\
\hline Sufficiently trained in using the FTT & $77 \%$ & \\
\hline $\begin{array}{l}\text { Interested in additional training in using } \\
\text { the FTT }\end{array}$ & $27 \%$ & $28 \%^{d}$ \\
\hline Resistance to change & & $39 \%^{e}$ \\
\hline Lack of experience & & $11 \%^{e}$ \\
\hline External barriers & & \\
\hline
\end{tabular}




\begin{tabular}{|l|c|c|}
\hline The FTT is time-consuming & $16 \%$ & \\
\hline The FTT is highly demanding & $7 \%$ & \\
\hline Children are able to reach their maximal & $32 \%$ & \\
effort & disagreed & \\
\hline Treadmill ergometer is available & $100 \%$ & $73 \%$ \\
& & \\
\hline
\end{tabular}

${ }^{a}$ FTT $=$ Fitkids Treadmill Test.

${ }^{b}$ Based on $n=48$

${ }^{c}$ Based on $n=89$

${ }^{d}$ Based on $n=50$

${ }^{e}$ Based on $n=44$ 